\title{
INVARIANCE OF SOLUTIONS TO INVARIANT NONPARAMETRIC VARIATIONAL PROBLEMS
}

\author{
BY
}

JOHN E. BROTHERS

\begin{abstract}
Let $f$ be a weak solution to the Euler-Lagrange equation of a convex nonparametric variational integral in a bounded open subset $D$ of $\mathbf{R}^{n}$. Assume the boundary $B$ of $D$ to be rectifiable. Let $D$ be a compact connected Lie group of diffeomorphisms of a neighborhood of $D \cup B$ which leave $D$ invariant and assume the variational integral to be $G$-invariant. Conditions are formulated which imply that if $f$ is continuous on $D \cup B$ and $f \circ g|B=f| B$ for $g \in G$ then $f \circ g=f$ for every $g \in G$. If the integrand $L$ is strictly convex then $f$ can be shown to have a local uniqueness property which implies invariance. In case $L$ is not strictly convex the graph $T_{f}$ of $f$ in $\mathbf{R}^{n} \times \mathbf{R}$ is interpreted as the solution to an invariant parametric variational problem, and invariance of $T_{f}$, hence of $f$, follows from previous results of the author. For this purpose a characterization is obtained of those nonparametric integrands on $\mathbf{R}^{n}$ which correspond to a convex positive even parametric integrand on $\mathbf{R}^{n} \times \mathbf{R}$ in the same way that the nonparametric area integrand corresponds to the parametric area integrand.
\end{abstract}

1. Introduction. Let $L: \mathbf{R}^{n} \times \mathbf{R} \times\left(\mathbf{R}^{n}\right)^{*} \rightarrow \mathbf{R}$ be a positive nonparametric variational integrand of class 1 which is convex (but not necessarily strictly convex) with respect to the third variable $\xi \in\left(\mathbf{R}^{n}\right)^{*}$. Let $D$ be a bounded open subset of $\mathbf{R}^{n}$ with boundary $B$ and $f: D \cup B \rightarrow \mathbf{R}$ be a continuous function such that $f \mid D$ is locally Lipschitzian, and assme $f$ to be a weak solution in $D$ to the Euler-Lagrange equation

$$
\operatorname{div}\left(D_{\xi} L\right)_{f}=\left(D_{z} L\right)_{f}
$$

(Elements of the domain of $L$ are denoted $(x, z, \xi)$, and $\left(D_{z} L\right)_{f}(x)=$ $D_{z} L(x, f(x), d f(x))$, etc.)

If $g$ is a diffeomorphism of $D$ onto $D$ then $L$ is called a $g$-invariant integrand provided

$$
L\left(g(x), z, D g(x)^{-1 *}(\xi)\right) J g(x)=L(x, z, \xi) \quad \text { for }(x, z, \xi) \in D \times \mathbf{R} \times\left(\mathbf{R}^{n}\right)^{*},
$$

where $J g(x)=|\operatorname{det} D g(x)|$. This implies that $f \circ g$ is a weak solution in $D$ to the Euler-Lagrange equation and also that

$$
\int_{D} L_{\phi} d \mathfrak{L}^{n}=\int_{D} L_{\phi \circ g} d \mathfrak{L}^{n}
$$

whenever $\phi: D \rightarrow \mathbf{R}$ is locally Lipschitzian. For example, if $g$ is an isometry of $\mathbf{R}^{n}$ and $L$ can be expressed as a function of $z$ and $|\xi|$ then $L$ is $g$-invariant.

Received by the editors November 1, 1979.

AMS (MOS) subject classifications (1970). Primary 49B25; Secondary 35J20, 49F20, 49F22.

Key words and phrases. Nonparametric integrand, parametric integrand, invariant integrand, EulerLagrange equation, weak solution. 
Let $G$ be a compact connected Lie group of diffeomorphisms of a neighborhood $U$ of $D \cup B$ onto $U$ which leave $D$ invariant, and assume $L$ to be a $g$-invariant integrand for $g \in G$. Our goal is to establish conditions on $L$ and $D$ under which it will be true that if

$$
f \circ g|B=f| B \quad \text { whenever } g \in G
$$

then

$$
f \circ g=f \quad \text { whenever } g \in G .
$$

For example, if $G$ is the group of rotations of $\mathbf{R}^{n}$ then $B$ will be the union of a finite family of $(n-1)$-dimensional spheres centered at the origin, and our theorem will imply that if $f$ is constant on each of these spheres then $f$ can be expressed as a function of $|x|$.

Inasmuch as $G$ is generated by a neighborhood of the identity, invariance would be implied by local uniqueness of $f$. Such a result is obtained in $\$ 3$ for the case where $L$ is strictly convex in $\xi$ and $D_{\xi} L$ is bounded.

In case $L$ is not strictly convex in $\xi$ our method is to interpret the graph $T_{f}$ of $f$ in $\mathbf{R}^{n} \times \mathbf{R}$ as a solution to an invariant parametric variational problem and infer invariance of $T_{f}$, hence of $f$, from an extension of the main result of [B]. For this purpose we are led in $\$ 4$ to characterize those integrands $L$ which correspond to a convex positive even parametric integrand $\Psi_{L}$ on $\mathbf{R}^{n} \times \mathbf{R}$ in the same way that the nonparametric area integrand corresponds to the parametric area integrand. We then use Hilbert's invariant integral as generalized in [F1, 5.4.18] to show in $\$ 5$ that $T_{f}$ is $\Psi_{L}$ minimizing with respect to suitable subsets of $D \cup B \times \mathbf{R}$.

Nonparametric integrands which arise from parametric integrands have been considered for $n=2$ by Finn and by Jenkins and Serrin as well as other investigators. (See [GT, p. 377].) The corresponding Euler-Lagrange equations are termed "of mean curvature type". Our conditions on $L$ are quite different from those of the above authors, which depend on the assumption $n=2$, but are related to those discussed in Chapter 15 of [GT]. Insofar as we know, ours is the first attempt at formulating conditions on $L$ which imply the existence of a parametric integrand giving rise to $L$.

In $\S 6$ we establish conditions on a $G$-invariant integrand $L$ which will imply that $\Psi_{L}$ has an invariance property similar to the one introduced in [B], thereby allowing us to apply an extension of the main result of [B]. These conditions are satisfied by a large class of integrands; for example, if $G$ is a group of isometries of $\mathbf{R}^{n}$ then $L$ will be of this type if it is of the form $\mathcal{L}(|\xi|)$ where $\mathcal{L}$ is a strictly increasing function. The conditions placed on $D$ in order to obtain the invariance theorem are satisfied if either $B$ is a submanifold of class 2 or $B$ is the union of a countable family of submanifolds of class 1 (of various dimensions) and $D$ is convex or, more generally, star-shaped.

This work was partially supported by a research grant from the National Science Foundation.

2. Preliminaries. The purpose of this section is to fix basic notation and terminology and to establish the general hypotheses which will be used in the 
paper. Notation and terminology which are not explained below may be found in [F1] or [B, §2]; see especially the glossary of notation on pp. 669-671 of [F1].

2.1. Throughout the paper $L, D, B$ and $f$ will be as in the first paragraph of $\S 1$. In addition $B$ is assumed to be rectifiable in dimension $n-1$ [F1, 3.2.14].

The projection of $\mathbf{R}^{n} \times \mathbf{R}$ onto $\mathbf{R}^{n}$ will be denoted by $p$ as will also the corresponding maps of the exterior powers $\bigwedge_{k}\left(\mathbf{R}^{n} \times \mathbf{R}\right)$ which are induced by $p$. When convenient we will identify $\mathbf{R}^{n}$ with the subspace $\mathbf{R}^{n} \times\{0\}$ of $\mathbf{R}^{n} \times \mathbf{R}$ and similarly identify $\bigwedge_{k}\left(\mathbf{R}^{n}\right)$ with $\bigwedge_{k}\left(\mathbf{R}^{n} \times\{0\}\right) \subset \bigwedge_{k}\left(\mathbf{R}^{n} \times \mathbf{R}\right)$. We identify $\mathbf{R}^{n} \times$ $\mathbf{R}$ with $\mathbf{R}^{n+1}$ and denote the standard basis vectors of $\mathbf{R}^{n+1}$ by $e_{1}, \ldots, e_{n+1}$.

Whenever $f_{0}: D \rightarrow \mathbf{R}$ define $\Gamma_{f_{0}}: D \rightarrow \mathbf{R}^{n+1}$ by the formula $\Gamma_{f_{0}}(x)=\left(x, f_{0}(x)\right)$.

We denote by $A$ the $n$-dimensional nonparametric area integrand defined by the formula

$$
A(\xi)=\left(1+|\xi|^{2}\right)^{1 / 2} \text { for } \xi \in\left(\mathbf{R}^{n}\right)^{*} .
$$

Suppose $H: U \rightarrow \mathbf{R}$ where $U$ is an open subset of $\mathbf{R}^{n} \times \mathbf{R}^{m}$ and the elements of $\mathbf{R}^{n} \times \mathbf{R}^{m}$ are denoted $(x, y)$. If $H$ is differentiable at $\left(x_{0}, y_{0}\right) \in U$ with (Fréchet) differential $D H\left(x_{0}, y_{0}\right) \in\left(\mathbf{R}^{n} \times \mathbf{R}^{m}\right)^{*}$ then one defines the partial differential $D_{x} H\left(x_{0}, y_{0}\right) \in\left(\mathbf{R}^{n}\right)^{*}$ by the formula $D_{x} H\left(x_{0}, y_{0}\right)=D H_{y_{0}}\left(x_{0}\right)$, where $H_{y}=H(\cdot, y)$. $D_{y} H\left(x_{0}, y_{0}\right)$ is defined similarly. We also denote $D_{x^{i}} H=\partial H / \partial x^{i}$, etc. Higher order partial differentials such as $D_{x}^{2} H$ are defined analogously.

By $f$ being a weak solution in $D$ to the Euler-Lagrange equation we mean that

$$
\int_{D}\left\langle\left(D_{\xi} L\right)_{f}, d \phi\right\rangle+\left(D_{z} L\right)_{f} \phi d L^{n}=0 \quad \text { whenever } \phi \in \mathscr{D}^{0}(D) .
$$

We remark that this equation also holds if $\phi$ is merely assumed to be a Lipschitz function with compact support in $D$. To verify this one first smooths $\phi$ (see for example [F1, 4.1.2]) and then applies Lebesgue's bounded convergence theorem.

2.2. For use throughout the paper we assume $f$ to be embedded in a field of extremals as follows:

There exist $\rho_{0}>0$ and a continuous function $F: D \cup B \times\left(-\rho_{0}, \rho_{0}\right) \rightarrow \mathbf{R}$ with the following five properties:

(i) $F(x, 0)=f(x)$ for $x \in D \cup B$.

(ii) $F \mid D \times\left(-\rho_{0}, \rho_{0}\right)$ is locally Lipschitzian as is also $D_{\rho} F \mid D \times\left(-\rho_{0}, \rho_{0}\right)$.

(iii) $F_{x}=F(x, \cdot)$ is strictly increasing whenever $x \in D \cup B$.

(iv) For every $\rho \in\left(-\rho_{0}, \rho\right), F_{\rho}=F(\cdot, \rho)$ is a weak solution in $D$ to the Euler-Lagrange equation.

Now define $\Phi: D \cup B \times\left(-\rho_{0}, \rho_{0}\right) \rightarrow \mathbf{R}^{n+1}$ so that $\Phi(x, \rho)=(x, F(x, \rho)) . \Phi$ is a homeomorphism and by invariance of domain $\Phi_{0}=\Phi \mid D \times\left(-\rho_{0}, \rho_{0}\right)$ maps $D \times$ $\left(-\rho_{0}, \rho_{0}\right)$ onto an open subset $\Omega$ of $\mathbf{R}^{n+1}$.

By Rademacher's theorem [F1, 3.1.6] $F$ is differentiable at almost all $(x, \rho) \in D$ $\times\left(-\rho_{0}, \rho_{0}\right)$. Denoting $(x, z)=\Phi(x, \rho)$ we define

$$
\nu_{L}(x, z)=\left(-\left(D_{\xi} L\right)_{F_{\rho}}(x), L_{F_{p}}(x)-\left\langle\left(D_{\xi} L\right)_{F_{p}}(x), d F_{\rho}(x)\right\rangle\right) \in \mathbf{R}^{n+1} .
$$

Inasmuch as $\Phi_{0}$ is locally Lipschitzian, $\nu_{L}(x, z)$ is defined for almost all $(x, z) \in \Omega$ and $\nu_{L}$ is $\mathcal{L}^{n+1}$ measurable. 
Finally, we assume that

(v) $\Phi_{0}^{-1}$ is locally Lipschitzian.

Denote by $\delta_{0}$ the smaller of the positive numbers

$$
\inf _{x \in D \cup B} \sup _{0<\rho<\rho_{0}} F_{\rho}(x)-f(x), \quad \inf _{x \in D \cup B} \sup _{0<\rho<\rho_{0}} f(x)-F_{-\rho}(x) .
$$

In case $L$ does not depend on $z$ we can let $F(x, \rho)=f(x)+\rho$, hence $\rho_{0}=\infty$. More generally, if $f(D \cup B)$ lies in an open interval $I$ such that there exists a function of class $2 H: I \times\left(-\rho_{0}, \rho_{0}\right) \rightarrow \mathbf{R}$ with the following properties then it is not difficult to show that the function defined by the formula $F(x, \rho)=H(f(x), \rho)$ will satisfy (i), (ii), (iii):

(a) $H(z, 0)=z$ for $z \in I$.

(b) $D_{z} H(z, \rho)>0$ and $D_{\rho} H(z, \rho)>0$ for $(z, \rho) \in I \times\left(-\rho_{0}, \rho_{0}\right)$.

(c) $L\left(x, H_{\rho}(z), H_{\rho}^{\prime}(z) \xi\right)=L(x, z, \xi)$ for $x \in D \cup B, \rho \in\left(-\rho_{0}, \rho_{0}\right), z \in I, \xi \in$ $\left(\mathbf{R}^{n}\right)^{*}$.

For example, if $k$ is an even positive integer and $L(x, z, \xi)=\mathscr{L}\left(x, z^{k}|\xi|\right)$ then (c) will be satisfied if $H_{\rho}^{\prime}>0$ and $H_{\rho}(z)^{k} H_{\rho}^{\prime}(z)=z^{k}$; consequently, the function $H$ defined by the formula $H(z, \rho)=\left(z^{k+1}+\rho\right)^{1 /(k+1)}$ will satisfy (a), (b), (c) provided $0 \notin I$ and $\rho_{0}$ is chosen so small that

$$
\left(-\rho_{0}^{1 /(k+1)}, \rho_{0}^{1 /(k+1)}\right) \cap I=\varnothing .
$$

We remark in closing that it is shown in $[M, \S 1.6]$ that $F$ exists provided $L, B$ and $f$ are sufficiently smooth, $D_{\xi}^{2} L$ is positive definite and the second variation of $\int_{D} L_{f} d \mathcal{L}^{n}$ is positive. However, this last condition already implies the uniqueness property we are seeking.

2.3. In $\S \S 4,5$ and 6 we will consider the following conditions; let $L$ be of class 2:

For each compact subset $K$ of $\mathbf{R}^{n+1}$ there exist constants $C_{K}$ and $c_{K}>0$ such that whenever $(x, z, \xi) \in K \times\left(\mathbf{R}^{n}\right)^{*}$

(A) $L(x, z, \xi)=L(x, z,-\xi)$,

(B) $L(x, z, \xi) \geqslant c_{K} A(\xi)$

(C) $\left|D_{\xi}^{2} L(x, z, \xi)\right| \leqslant C_{K} A(\xi)^{-1}$,

(D) $\left|D_{\xi}^{2} L(x, z, \xi)(\xi, \eta)\right|<C_{K} A(\xi)^{-2}|\xi||\eta|$ for $\eta \in\left(\mathbf{R}^{n}\right)^{*}$,

(E) $D_{\xi}^{2} L(x, z, \xi)(\xi, \xi) \leqslant C_{K} A(\xi)^{-3}|\xi|^{2}$,

(F) $L(x, z, 0) \geqslant \int_{0}^{\infty} t D_{\xi}^{2} L(x, z, t \xi)(\xi, \xi) d t$.

Note that in case $L=A$ these conditions are satisfied with $C_{K}=1$ (and equality in $(\mathrm{F})$ for $\xi \neq 0$ ).

2.4. In $\S \S 5$ and 6 we will assume that $L$ satisfies conditions $2.3(\mathrm{~A})-(\mathrm{E})$ together with the following: There exists a constant $C$ such that whenever $(x, z, \xi) \in \Omega \times$ $\left(\mathbf{R}^{n}\right)^{*}$

$(\mathrm{E})_{\Omega} D_{\xi}^{2} L(x, z, \xi)(\xi, \xi) \leqslant C A(\xi)^{-3}|\xi|^{2}$,

$(\mathrm{F})_{\Omega} L(x, z, 0) \geqslant C$.

Note that in case $\Omega$ is bounded or $L$ does not depend on $z$ (in which case $\left.\rho_{0}=\infty\right),(\mathrm{E})_{\Omega}$ is implied by $(\mathrm{E})$. Further, since $D$ is bounded and $L(x, z, \cdot)$ takes on its absolute minimum at $\xi=0$ because of $(A)$, in either of these cases $(F)_{\Omega}$ can 
be realized by replacing $L$ by $L+C$, which obviously does not affect $f$. Finally, we observe that $(\mathrm{E})_{\Omega}$ and $(\mathrm{F})_{\Omega}$ together imply $(\mathrm{F})$.

2.5. In $\S \S 5$ and 6 we will assume that for each $i=1,2, \ldots$ there exist open sets $D_{i}, U_{i}$ such that

$$
\text { Closure } D_{i} \subset D, \quad D \cup B \subset U_{i}, \quad D_{i} \subset D_{i+1}, \quad D=\bigcup_{j=1}^{\infty} D_{j},
$$

and an isotopic deformation of class $1 h_{i}: J_{i} \times U_{i} \rightarrow U_{i}$ with the following properties: $J_{i}$ is an open interval, $0 \in J_{i}, h_{i, 0}$ is the identity, and for each $0<t \in J_{i}$, $h_{i, t}=h_{i}(t, \cdot)$ is a diffeomorphism of class 1 of $U_{i}$ for which

$$
h_{i, t} \mid D_{i}=\mathbf{1}_{D_{i}} \text { and } h_{i, t}(D \cup B) \subset D \text {. }
$$

\section{Uniqueness.}

3.1. Lemma. Suppose $\nu_{L}(x, z)$ is defined, $(x, z)=\Phi(x, \rho)$. Then

(i) $\left\langle\nu_{L}\left(x, F_{\rho}(x)\right),\left(-d F_{\rho}(x), 1\right)\right\rangle=L\left(x, F_{\rho}(x), d F_{\rho}(x)\right)$.

(ii) $\left\langle\nu_{L}(x, z),(-\xi, 1)\right\rangle \leqslant L(x, z, \xi)$ for $\xi \in\left(\mathbf{R}^{n}\right)^{*}$; if $L(x, z, \cdot)$ is strictly convex then equality can hold only for $\xi=d F_{\rho}(x)$.

Furthermore, if $\eta \in \mathscr{D}^{0}(\Omega)$ then

(iii) $\int_{\Omega}\left\langle\nu_{L}, d \eta\right\rangle d \mathcal{L}^{n+1}=0$.

Proof. (i) is obvious and (ii) is equivalent to the convexity hypothesis on $L$.

For the proof of (iii) we denote $\Omega_{0}=D \times\left(-\rho_{0}, \rho_{0}\right)$, define

$$
\nu(x, \rho)=\left(-\left(D_{\xi} L\right)_{F_{p}}(x) D_{\rho} F(x, \rho), L_{F_{p}}(x)\right)
$$

whenever $F$ is differentiable at $(x, \rho)$, and with the aid of [F1, 3.2.5] compute

$$
\begin{aligned}
D \Phi(x, \rho) \nu(x, \rho) & =J \Phi(x, \rho) \nu_{L} \circ \Phi(x, \rho) \\
\int_{\Omega}\left\langle\nu_{L}, d \eta\right\rangle d \mathcal{L}^{n+1} & =\int_{\Omega_{0}}\left\langle\nu_{L} \circ \Phi, d \eta \circ \Phi\right\rangle J \Phi d \mathcal{L}^{n+1} \\
& =\int_{\Omega_{0}}\langle\nu, d(\eta \circ \Phi)\rangle d L^{n+1} .
\end{aligned}
$$

For each $\varepsilon>0$ we next smooth $F$ (see for example [F1, 4.1.2]) to obtain approximating functions $F^{e} \in \mathscr{D}^{0}\left(\mathbf{R}^{n+1}\right)$ and define $\nu^{e}$ in the same way as $\nu$ with $F$ replaced by $F^{\varepsilon}$. Now as $\varepsilon \rightarrow 0^{+}, F^{e} \rightarrow F$ uniformly on spt $\eta \circ \Phi$ and $D F^{e}(x, \rho) \rightarrow$ $D F(x, \rho)$ for almost all $(x, \rho) \in \Omega_{0}$; moreover, $\left|D F^{e}\right|$ is bounded on spt $\eta \circ \Phi$ by a Lipschitz constant for $F \mid$ spt $\eta \circ \Phi$. Consequently, Lebesgue's bounded convergence theorem implies that

$$
\lim _{\varepsilon \rightarrow 0^{+}} \int_{\Omega_{0}}\left\langle\nu^{\varepsilon}, d(\eta \circ \Phi)\right\rangle d \mathscr{L}^{n+1}=\int_{\Omega_{0}}\langle\nu, d(\eta \circ \Phi)\rangle d \mathscr{L}^{n+1} .
$$

On the other hand, in view of the fact that spt $\eta \circ \Phi \subset \Omega_{0}$ we can for each $\varepsilon>0$ use Fubini's theorem and integration by parts twice to obtain 


$$
\begin{aligned}
\int_{\Omega_{0}}\left\langle\nu^{\varepsilon}, d(\eta \circ \Phi)\right\rangle d L^{n+1}=-\int_{\Omega_{0}}\left(\operatorname{div} \nu^{\varepsilon}\right) \eta \circ \Phi d \mathcal{L}^{n+1} \\
=\int_{\Omega_{0}}\left\langle\left(D_{\xi} L\right)_{F_{\rho}^{e}}(x), d \psi_{\rho}^{\varepsilon}(x)\right\rangle+\left(D_{z} L\right)_{F_{\rho}^{e}}(x) \psi_{\rho}^{\varepsilon}(x) d L^{n+1}(x, \rho)
\end{aligned}
$$

where $\psi_{\rho}^{\varepsilon}=\left(D_{\rho} F^{\varepsilon}\right)_{\rho}(\eta \circ \Phi)_{\rho}$. Finally, we let $\varepsilon \rightarrow 0^{+}$and again use Lebesgue's theorem and Fubini's theorem to infer that

$$
\begin{aligned}
\int_{\Omega_{0}}\langle\nu, d(\eta \circ \Phi)\rangle d \mathcal{L}^{n+1} \\
\quad=\int_{\rho_{0}}^{\rho_{0}} \int_{D}\left\langle\left(D_{\xi} L\right)_{F_{\rho}}(x), d \psi_{\rho}(x)\right\rangle+\left(D_{z} L\right)_{F_{\rho}}(x) \psi_{\rho}(x) d \mathcal{L}^{n} x d \mathcal{L}^{1} \rho
\end{aligned}
$$

where $\psi_{\rho}=\left(D_{\rho} F\right)_{\rho}(\eta \circ \Phi)_{\rho}$. But $\psi_{\rho}$ is Lipschitzian by 2.2 hence the inner integral vanishes for almost all $\rho$.

3.2. Lemma. Assume that $\left|D_{\xi} L(x, z, \xi)\right| \leqslant C$ for each $(x, z, \xi) \in \Omega \times\left(\mathbf{R}^{n}\right)^{*}$ and $f_{0}$ : $D \cup B \rightarrow \mathbf{R}$ is continuous with $f_{0} \mid D$ locally Lipschitzian and

$$
\left|f_{0}(x)-f(x)\right|<\delta_{0} \quad \text { for } x \in D \text {. }
$$

Then

$$
\int_{D} L_{f} d \mathcal{L}^{n} \leqslant \int_{D} L_{f_{0}} d \mathcal{L}^{n}+4 C \sup _{B}\left|f-f_{0}\right| \mathcal{F C}^{n-1}(B)
$$

and $\int_{D} L_{f} d \mathcal{L}^{n}<\infty$

Proof. To obtain the second assertion from the first extend $f$ to a continuous function on $\mathbf{R}^{n}$, select $f_{0} \in \mathcal{E}^{0}\left(\mathbf{R}^{n}\right)$ such that $\left|f_{0}(x)-f(x)\right|<\delta_{0}$ for $x \in D$, and observe that $\int_{D} L_{f_{0}} d \mathcal{L}^{n}<\infty$.

The remainder of the proof was suggested by a technique employed in $[\mathbf{H}]$.

For $x \in \mathbf{R}^{n}$ denote by $\delta(x)$ the distance from $x$ to $\mathbf{R}^{n} \sim D$. $\delta$ has Lipschitz constant 1 hence $\delta$ is differentiable at $x$ with $J \delta(x)=|D \delta(x)|<1$ for almost all $x$. Whenever $\varepsilon>0$ application of the coarea formula [F1, 3.2.11] yields

$$
\mathcal{L}^{n}\{x: 0<\delta(x)<\varepsilon\} \geqslant \int_{0}^{\varepsilon} \mathcal{H}^{n-1}\{x: \delta(x)=t\} d t .
$$

Inasmuch as $B$ is rectifiable,

$$
\lim _{\varepsilon \rightarrow 0^{+}} \varepsilon^{-1} \varrho^{n}\{x: 0<\delta(x)<\varepsilon\}=\mathcal{H}^{n-1}(B)<\infty
$$

by [F1, 3.2.29] hence with the aid of [F1, 3.2.22] we can select a decreasing sequence of positive numbers $\varepsilon_{i}$ converging to 0 such that for each $i=1,2, \ldots$

$$
\mathcal{F}^{n-1}\left\{x: \delta(x)=\varepsilon_{i}\right\}<2 \mathcal{H}^{n-1}(B)
$$

and $B_{i}=\left\{x: \delta(x)=\varepsilon_{i}\right\}$ is $\left(\mathcal{H}^{n-1}, n-1\right)$ rectifiable. Denote $\Delta_{i}=\left\{x: \delta(x)>\varepsilon_{i}\right\}$; we have $B_{i} \supset$ boundary $\Delta_{i}$. Also denote $Q_{i}=\mathbf{E}^{n} L \Delta_{i} \in \Re_{n}\left(\mathbf{R}^{n}\right)$. Then $\mathbf{M}\left(\partial Q_{i}\right)<\infty$ by [F1, 4.5.11 and 2.10.6] and so $\left\|\partial Q_{i}\right\|<\mathcal{F}^{n-1}\left\llcorner B_{i}\right.$ by [F1, 4.5.6]. Furthermore, $\partial Q_{i} \in \Re_{n-1}\left(\mathbf{R}^{n}\right)$ by $[\mathbf{F 1}, 4.2 .16]$. 
Next consider continuous functions $f_{1}, f_{2}: D \cup B \rightarrow \mathbf{R}$ such that $f_{1} \mid D$ and $f_{2} \mid D$ are locally Lipschitzian, define the affine homotopy $h: \mathbf{R} \times D \rightarrow D \times \mathbf{R}$ by

$$
h(t, x)=\left(x, t f_{2}(x)+(1-t) f_{1}(x)\right),
$$

fix $i$, and (keeping [F1, 4.1.14] in mind) use [F1, 4.1.9] to compute

$$
\Gamma_{f_{2} \#} Q_{i}-\Gamma_{f_{1} \#} Q_{i}=\partial h_{\#}\left([0,1] \times Q_{i}\right)+h_{\#}\left([0,1] \times \partial Q_{i}\right) .
$$

Using [F1, 4.1.28 and 4.1.30] we estimate

$$
\begin{aligned}
\mathbf{M} h_{\#}\left([0,1] \times \partial Q_{i}\right) & \leqslant \sup _{B_{i}}\left|f_{2}-f_{1}\right| \mathcal{F C}^{n-1}\left(B_{i}\right) \\
& \leqslant 2 \sup _{B_{i}}\left|f_{2}-f_{1}\right| \mathcal{F C}^{n-1}(B) .
\end{aligned}
$$

Since $f_{2}-f_{1}$ is uniformly continuous, for $i$ sufficiently large

$$
\left|f_{2}(x)-f_{1}(x)\right| \leqslant 2 \sup _{B}\left|f_{2}-f_{1}\right| \quad \text { whenever } x \in B_{i}
$$

and we conclude that

$$
\limsup _{i \rightarrow \infty} \mathbf{M} h_{\#}\left([0,1] \times \partial Q_{i}\right)<4 \sup _{B}\left|f_{2}-f_{1}\right| \mathcal{F}^{n-1}(B) .
$$

Choose smoothing functions $\phi^{e}, \varepsilon>0$, for $\mathbf{R}^{n+1}$ and, fixing $i$, infer the existence for each $\varepsilon>0$ of $\eta^{\varepsilon} \in \mathscr{D}^{0}(\Omega)$ such that

$$
\mathbf{E}^{n+1}\left\llcorner\eta^{\varepsilon}=h_{\#}\left([0,1] \times Q_{i}\right) * \phi^{e} .\right.
$$

Assume that for $\alpha=1,2, \Gamma_{f_{\alpha}}(x) \in \Omega$ for $x \in D$. Referring to [F1, 1.5.2 and 4.1.7] and applying 3.1(iii) we have

$$
\begin{aligned}
\partial\left(\mathbf{E}^{n+1}\left\llcorner\eta^{e}\right)\left(\mathbf{D}_{1} \nu_{L}\right)\right. & =-\mathbf{E}^{n+1}\left\llcorner d \eta^{e}\left(\mathbf{D}_{1} \nu_{L}\right)\right. \\
& =(-1)^{n+1} \int_{\Omega}\left\langle\nu_{L}, d \eta^{e}\right\rangle d \mathscr{L}^{n+1}=0,
\end{aligned}
$$

hence

$$
\partial h_{\#}\left([0,1] \times Q_{i}\right)\left(\mathbf{D}_{1} \nu_{L}^{e}\right)=0 \text { for } \varepsilon>0,
$$

where $\nu_{L}^{e}=\phi^{\varepsilon} * \nu_{L}$. Applying this in (1) and again referring to [F1, 4.1.9] we obtain

$$
\Gamma_{f_{2} \#} Q_{i}\left(\mathbf{D}_{1} v_{L}^{\varepsilon}\right)-\Gamma_{f_{1} \#} Q_{i}\left(\mathbf{D}_{1} \nu_{L}^{e}\right)<C \mathbf{M} h_{\#}\left([0,1] \times \partial Q_{i}\right) .
$$

Next observe that $\nu_{L}^{e}(x, z) \rightarrow \nu_{L}(x, z)$ as $\varepsilon \rightarrow 0^{+}$for almost all $(x, z) \in \Omega$. We apply the coarea formula [F1, 3.2.11] to the functions $H_{\alpha}: D \times \mathbf{R} \rightarrow \mathbf{R}, \alpha=1,2$, defined by the formula $H_{\alpha}(x, z)=f_{\alpha}(x)+z$ to obtain $z_{j} \in \mathbf{R}, j=1,2, \ldots$, such that

$$
\begin{aligned}
z_{j} & \rightarrow 0 \text { as } j \rightarrow \infty \quad \text { and } \\
\nu_{L}^{e}\left(x, f_{\alpha}(x)+z_{j}\right) & \rightarrow \nu_{L}\left(x, f_{\alpha}(x)+z_{j}\right) \quad \text { as } \varepsilon \rightarrow 0^{+}
\end{aligned}
$$

for almost all $x \in D$.

Recalling [F1, 4.1.14 and 1.5.2] we denote $T_{0}=\Gamma_{f_{0} \#} Q_{i}$, fix $x \in \Delta_{i}$ such that $D f_{0}(x)$ exists, and compute 


$$
\begin{aligned}
& \left|D \Gamma_{f_{0}}(x)\right|=A_{f_{0}}(x), \\
& A_{f_{0}}(x) \vec{T}_{0}\left(x, f_{0}(x)\right)=D_{x^{1}} \Gamma_{f_{0}}(x) \wedge \cdots \wedge D_{x^{n}} \Gamma_{f_{0}}(x) \\
& =e_{1} \wedge \cdots \wedge e_{n}+\sum_{i=1}^{n}(-1)^{n-i} D_{x^{i}} f_{0}(x) e_{1} \wedge \\
& \cdots \wedge \hat{e}_{i} \wedge \cdots \wedge e_{n} \wedge e_{n+1}, \\
& A_{f_{0}}(x) \mathbf{D}_{n} \vec{T}_{0}\left(x, f_{0}(x)\right)=(-1)^{n}\left(-d f_{0}(x), 1\right) .
\end{aligned}
$$

Observing that

$$
\left\langle\nu_{L}^{\varepsilon}, \mathbf{D}_{n} \vec{T}_{0}\right\rangle=(-1)^{n}\left\langle\vec{T}_{0}, \mathbf{D}_{1} \nu_{L}^{\varepsilon}\right\rangle
$$

and referring to [F1, 4.1.30 and 3.2.20] we compute

$$
\Gamma_{f_{0} \#} Q_{i}\left(\mathbf{D}_{1} \nu_{L}^{\varepsilon}\right)=\int_{\Delta_{i}}\left\langle\nu_{L}^{\varepsilon}\left(x, f_{0}(x)\right),\left(-d f_{0}(x), 1\right)\right\rangle d \mathcal{L}^{n} x .
$$

Applying this with $f_{0}$ replaced by $f_{\alpha}+z_{j}$ we first let $\varepsilon \rightarrow 0^{+}$and then let $j \rightarrow \infty$ and conclude with the aid of Lebesgue's bounded convergence theorem and (4) that

$$
\begin{aligned}
\int_{\Delta_{i}}\left\langle\nu_{L}\left(x, f_{2}(x)\right),\left(-d f_{2}(x), 1\right)\right\rangle d L^{n} x \\
\quad-\int_{\Delta_{i}}\left\langle\nu_{L}\left(x, f_{1}(x)\right),\left(-d f_{1}(x), 1\right)\right\rangle d \mathcal{L}^{n} x<C M h_{\#}\left([0,1] \times \partial Q_{i}\right) .
\end{aligned}
$$

Finally, 3.1 implies that for each measurable set $A$ with compact closure in $D$

$$
\int_{A}\left\langle\nu_{L}\left(x, f_{\alpha}(x)\right),\left(-d f_{\alpha}(x), 1\right)\right\rangle d \mathcal{L}^{n} x<\int_{A} L_{f_{\alpha}} d \mathcal{L}^{n}
$$

with equality if $f_{\alpha}=f$. Applying this in (7) with $f_{2}=f$ and $f_{1}=f_{0}$ we let $i \rightarrow \infty$ and refer to (2) to obtain our conclusion.

3.3. Corollary. In addition to the hypotheses of 3.2 assume $L(x, z, \cdot)$ to be strictly convex for each $(x, z) \in \Omega$. If $f_{0}|B=f| B$ and

$$
\int_{D} L_{f_{0}} d \mathcal{L}^{n}=\int_{D} L_{f} d \mathcal{L}^{n}
$$

then $f_{0}=f$.

Proof. Referring to (2), (7) and (8) in the proof of 3.2 we infer that

$$
\int_{D} L_{f} d \mathcal{L}^{n} \leqslant \limsup _{i \rightarrow \infty} \int_{\Delta_{i}}\left\langle\nu_{L}\left(x, f_{0}(x)\right),\left(-d f_{0}(x), 1\right)\right\rangle d \mathcal{L}^{n} x
$$

Assume there exists a compact subset $K$ of $D$ such that $\mathfrak{L}^{n}(K)>0$ and for $x \in K$

$$
d f_{0}(x) \neq d F_{\rho}(x) \text { where } f_{0}(x)=F_{\rho}(x) .
$$

Then by 3.1(ii)

$$
0<\delta=\int_{K} L_{f_{0}} d \mathcal{L}^{n}-\int_{K}\left\langle\nu_{L}\left(x, f_{0}(x)\right),\left(-d f_{0}(x), 1\right)\right\rangle d \mathcal{L}^{n} x
$$


hence for each $\Delta_{i} \supset K(8)$ implies that

$$
\int_{\Delta_{i}}\left\langle\nu_{L}\left(x, f_{0}(x)\right),\left(-d f_{0}(x), 1\right)\right\rangle d \varrho^{n} x \leqslant \int_{D} L_{f_{0}} d \varrho^{n}-\delta
$$

and we therefore conclude that for almost all $x \in D$

$$
d f_{0}(x)=d F_{\rho}(x) \quad \text { where } f_{0}(x)=F_{\rho}(x) .
$$

The latter equality is equivalent to $\Gamma_{f_{0}}(x)=\Phi(x, \rho)$, in view of which the former implies that $D \Gamma_{f_{0}}(x)\left(e_{i}\right)=D \Phi(x, \rho)\left(e_{i}\right)$ for $i=1, \ldots, n$. This implies that

$$
D\left(q \circ \Phi^{-1} \circ \Gamma_{f_{0}}\right)(x)=0
$$

since $\Phi^{-1}$ is locally Lipschitzian, where $q$ is the projection of $\mathbf{R}^{n+1}=\mathbf{R}^{n} \times \mathbf{R}$ on $\mathbf{R}$. It follows that $f_{0}=F_{\rho}$ with $\rho$ constant on each component of $D$. However, $F(x, \cdot)$ is strictly increasing for $x \in B$ hence we conclude that $\rho=0$.

3.4. Theorem. Let $G$ and $U$ be as in $\S 1$, and assume $L$ to be $G$-invariant. Assume also that $L(x, z, \cdot)$ is strictly convex for each $(x, z) \in \Omega$ and that $\left|D_{\xi} L(x, z, \xi)\right| \leqslant C$ for each $(x, z, \xi) \in \Omega \times\left(\mathbf{R}^{n}\right)^{*}$.

If $f \circ g \mid B=B$ whenever $g \in G$ then $f \circ g=f$ for every $g \in G$.

Proof. $G$ acts continuously by means of composition on the vector space of continuous real valued functions on $D \cup B$ with the uniform norm. The topology of $G$ is the compact-open topology hence there is a neighborhood $W$ of the identity such that whenever $g \in W$

$$
|f \circ g(x)-f(x)|<\delta_{0} \text { for } x \in D \cup B .
$$

Thus $f \circ g=f$ for $g \in W$ by 3.3 and also for arbitrary $g$ because $W$ generates $G$.

\section{Parametric integrands.}

4.1. LemMa. Assume conditions (A), (D), (F) in 2.3 hold.

(i) If $K$ is a compact subset of $\mathbf{R}^{n+1}$ then

$$
\left|D_{\xi} L(x, z, \xi)\right| \leqslant 2 C_{K} \quad \text { for }(x, z, \xi) \in K \times\left(\mathbf{R}^{n}\right)^{*} .
$$

(ii) $\left|\left\langle\nu_{L}(x, z),(-\xi, 1)\right\rangle\right| \leqslant L(x, z, \xi)$ for $(x, z, \xi) \in \Omega \times\left(\mathbf{R}^{n}\right)^{*}$.

Proof. Fix $(x, z) \in \mathbf{R}^{n+1}$ and denote $l=L(x, z, \cdot)$. (A) implies that $D l(0)=0$. Thus

$$
D l(\xi)(\eta)=\int_{0}^{1} D^{2} l(t \xi)(\xi, \eta) d t \quad \text { for } \eta \in\left(\mathbf{R}^{n}\right)^{*}
$$

and so (D) implies that $|D l(\xi)| \leqslant C_{K} \tan ^{-1}|\xi|$ if $(x, z) \in K$.

To verify (ii) first note that it will suffice to show that for $\xi_{0} \in\left(\mathbf{R}^{n}\right)^{*},\left|\tau_{\xi_{0}}(\xi)\right|<$ $l(\xi)$ where

$$
\tau_{\xi_{0}}(\xi)=l\left(\xi_{0}\right)+\left\langle D l\left(\xi_{0}\right), \xi-\xi_{0}\right\rangle
$$

Now $\tau_{\xi_{0}}(\xi) \leqslant l(\xi)$ because $l$ is convex. (A) implies that $\tau_{-\xi_{0}}(\xi)=\tau_{\xi_{0}}(-\xi)$ hence if we can show that $-\tau_{-\xi_{0}}(\xi) \leqslant \tau_{\xi_{0}}(\xi)$ it will follow that $-\tau_{\xi_{0}}(-\xi)<l(-\xi)$. But Taylor's 
theorem and (F) imply that

$$
\begin{aligned}
\frac{1}{2}\left[\tau_{-\xi_{0}}(\xi)+\tau_{\xi_{0}}(\xi)\right] & =l\left(\xi_{0}\right)+\left\langle D l\left(\xi_{0}\right),-\xi_{0}\right\rangle \\
& =l(0)-\int_{0}^{1} t D^{2} l\left(t \xi_{0}\right)\left(\xi_{0}, \xi_{0}\right) d t>0 .
\end{aligned}
$$

4.2. REMARK. 4.1(ii) is essential for the use of the parametric version of Hilbert's invariant integral in the proof of 5.3. Simple examples show that 2.3(A) alone does not imply (ii).

4.3. Lemma. Define $\Lambda: \mathbf{R}^{n+1} \times\left(\mathbf{R}^{n}\right)^{*} \times(\mathbf{R} \sim\{0\}) \rightarrow \mathbf{R}$ by

$$
\Lambda(x, z, \xi, \varepsilon)=L(x, z, \xi / \varepsilon)|\varepsilon| \text {. }
$$

Assume conditions 2.3(A)-(F) to hold. Then $\Lambda$ can be continuously extended to $\mathbf{R}^{n+1} \times\left(\mathbf{R}^{n}\right)^{*} \times \mathbf{R}$; moreover, the following are true:

(i) $\Lambda(x, z, \cdot, \cdot)$ is convex for $(x, z) \in \mathbf{R}^{n+1}$.

(ii) $\Lambda \mid K \times\left(\mathbf{R}^{n}\right)^{*} \times \mathbf{R}$ is Lipschitzian for each compact subset $K$ of $\mathbf{R}^{n+1}$.

(iii) $D_{\xi} \Lambda$ is continuous on $\mathbf{R}^{n+1} \times\left[\left(\mathbf{R}^{n}\right)^{*} \times \mathbf{R} \sim\{(0,0)\}\right]$.

(iv) The restriction of $D_{e} \Lambda$ to each component of $\mathbf{R}^{n+1} \times\left(\mathbf{R}^{n}\right)^{*} \times(\mathbf{R} \sim\{0\})$ can be continuously extended to the closure of its domain minus $\mathbf{R}^{n+1} \times\{(0,0)\}$. These extensions coincide at $(x, z, \xi, 0), \xi \neq 0$, if and only if equality holds in 2.3(F).

Proof. Fix $K$ as in (ii) and $(x, z) \in K$, and denote $l=L(x, z, \cdot), \lambda=$ $\Lambda(x, z, \cdot, \cdot)$. For $\varepsilon \neq 0$ we have $D_{\xi} \lambda(\xi, \varepsilon)=D l(\xi / \varepsilon)$ sign $\varepsilon$, and we use Taylor's theorem to obtain

$$
\begin{aligned}
D_{\varepsilon} \lambda(\xi, \varepsilon) & =[l(\xi / \varepsilon)-\langle D l(\xi / \varepsilon), \xi / \varepsilon\rangle] \operatorname{sign} \varepsilon \\
& =\left[l(0)-\int_{0}^{1 / \varepsilon} t D^{2} l(t \xi)(\xi, \xi) d t\right] \operatorname{sign} \varepsilon .
\end{aligned}
$$

Thus by 4.1 and $2.3(\mathrm{E})$

$$
\left|D_{\xi} \lambda(\xi, \varepsilon)\right| \leqslant 2 C_{K}, \quad\left|D_{\varepsilon} \lambda(\xi, \varepsilon)\right| \leqslant l(0)+C_{K} .
$$

These bounds imply that $\Lambda$ satisfies a Lipschitz condition on each of the sets

$$
K \times\left(\mathbf{R}^{n}\right)^{*} \times(0, \infty) \text { and } K \times\left(\mathbf{R}^{n}\right)^{*} \times(-\infty, 0)
$$

hence the restriction of $\Lambda$ to each of these sets can be extended to a Lipschitz function on the closure of its domain. Further, these functions must coincide on $K \times\left(\mathbf{R}^{n}\right)^{*} \times\{0\}$ because $\Lambda$ is an even function of $\varepsilon$ by $2.3(\mathrm{~A})$. Thus $\Lambda$ can be extended as asserted; note that (ii) also holds.

Again fixing $(x, z) \in K$ one next verifies that for $\varepsilon \neq 0$ the matrix of second partial derivatives of $\lambda$ at $(\xi, \varepsilon)$ is

$$
D^{2} \lambda(\xi, \varepsilon)=|\varepsilon|^{-3}\left[\begin{array}{cc}
\varepsilon^{2} D^{2} l & -|\varepsilon| B^{t} \\
-|\varepsilon| B & D^{2} l(\xi, \xi)
\end{array}\right]
$$

where $B$ is the $1 \times n$ matrix whose $i$ th entry is $D^{2} l\left(\xi, e_{i}\right)$ and the derivatives of $l$ are evaluated at $\xi / \varepsilon$. In particular, it follows from $2.3(\mathrm{C})$ and (D) that for each $i=1, \ldots, n$ all partial derivatives of $D_{\xi} \lambda$ at $(\xi, \varepsilon)$ are bounded by $C_{K} /|\xi|$. Consequently, for each $R>0, D_{\xi} \Lambda$ satisfies a Lipschitz condition on each of the 
sets

$$
\begin{gathered}
K\left[\left(\mathbf{R}^{n}\right)^{*} \times \mathbf{R} \cap\{(\xi, \varepsilon):|\xi| \geqslant R, \varepsilon<0\}\right], \\
K \times\left[\left(\mathbf{R}^{n}\right)^{*} \times \mathbf{R} \cap\{(\xi, \varepsilon):|\xi|>R, \varepsilon>0\}\right],
\end{gathered}
$$

hence can be continuously extended to their closures. However, these extensions coincide on $K \times\left(\mathbf{R}^{n}\right)^{*} \times\{0\}$ because $D_{\xi} \Lambda$ is an even function of $\varepsilon$. (iii) now follows easily.

To obtain the first part of (iv) use 2.3(D) and (E) in (3) and reason as above; to verify the second use $2.3(\mathrm{~A})$ and (1) to compute

$$
\begin{aligned}
\lim _{\varepsilon \rightarrow 0^{+}} D_{\varepsilon} \lambda(\xi, \varepsilon) & -\lim _{\varepsilon \rightarrow 0^{-}} D_{\varepsilon} \lambda(\xi, \varepsilon) \\
= & 2 \lim _{\varepsilon \rightarrow 0^{+}} D_{\varepsilon} \lambda(\xi, \varepsilon)=2\left(l(0)-\int_{0}^{\infty} t D^{2} l(t \xi)(\xi, \xi) d t\right) .
\end{aligned}
$$

Turning to (i) we note that for each $\xi, D^{2} l(\xi)$ is positive semidefinite because $l$ is convex. Further, for $\varepsilon \neq 0$ the last row of (3) is a linear combination of the remaining rows hence $\left[C\right.$, p. 188] implies that $D^{2} \lambda(\xi, \varepsilon)$ is positive semidefinite. With the help of Taylor's theorem we conclude that the restriction of $\lambda$ to each of the sets $\left(\mathbf{R}^{n}\right)^{*} \times[0, \infty),\left(\mathbf{R}^{n}\right)^{*} \times(-\infty, 0]$ is convex. Furthermore, if $\xi, \xi_{0} \in\left(\mathbf{R}^{n}\right)^{*}$ with $\xi \neq 0$ then by $2.3(\mathrm{~F})$, (iii) and (4)

$$
\begin{aligned}
\lim _{\varepsilon \rightarrow 0^{-}} D \lambda(\xi, \varepsilon)\left(\xi_{0}, 1\right) & =\lim _{\varepsilon \rightarrow 0^{-}} D_{\varepsilon} \lambda(\xi, \varepsilon)+\left\langle D_{\xi} \lambda(\xi, 0), \xi_{0}\right\rangle \\
& \leqslant \lim _{\varepsilon \rightarrow 0^{+}} D \lambda(\xi, \varepsilon)\left(\xi_{0}, 1\right) .
\end{aligned}
$$

Now any line in $\left(\mathbf{R}^{n}\right)^{*} \times \mathbf{R}$ which intersects both of the sets $\left(\mathbf{R}^{n}\right)^{*} \times(0, \infty)$ and $\left(\mathbf{R}^{n}\right)^{*} \times(-\infty, 0)$ can be written in the form $\gamma(t)=\left(t \xi_{0}+\xi_{1}, t\right), t \in \mathbf{R}$. Thus if $\xi_{1} \neq 0$ then $(\lambda \circ \gamma)^{\prime}$ is nondecreasing hence $\lambda \circ \gamma$ is convex. On the other hand, if $\xi_{1}=0$ then $\lambda \circ \gamma(t)=l\left(\xi_{0}\right)|t|$ hence again $\lambda \circ \gamma$ is convex. This, together with the above discussion, implies that $\lambda$ is convex.

4.4. Definition. Whenever $\alpha \in \bigwedge_{n}\left(\mathbf{R}^{n+1}\right)$ with $p(\alpha) \neq 0$ we define $\xi(\alpha) \in\left(\mathbf{R}^{n}\right)^{*}$ $=\bigwedge^{1}\left(\mathbf{R}^{n}\right)$ by the formula

$$
\xi(\alpha)=\left(\mathbf{D}_{n} p(\alpha)\right)^{-1} \mathbf{D}_{n-1} p(\alpha\llcorner d z)
$$

and $\Psi_{L}(x, z, \alpha)$ by the formula

$$
\Psi_{L}(x, z, \alpha)=L(x, z, \xi(\alpha))|p(\alpha)| \quad \text { for }(x, z) \in \mathbf{R}^{n+1} .
$$

Inasmuch as

$$
\Psi_{L}(x, z, \alpha)=\Lambda\left(x, z, \mathbf{D}_{n-1} p\left(\alpha\llcorner d z), \mathbf{D}_{n} p(\alpha)\right),\right.
$$

we see from 4.2 that $\Psi_{L}$ has a continuous extension to $\mathbf{R}^{n+1} \times \wedge_{n}\left(\mathbf{R}^{n+1}\right)$ if $L$ satisfies the conditions in 2.3, hence is a parametric integrand of degree $n$ [F1, 5.1.1]. 
4.5. TheORem. If $L$ satisfies conditions $2.3(\mathrm{~A})-(\mathrm{F})$ then $\Psi_{L}$ is a convex positive even parametric integrand with the following properties:

(a) $\Psi_{L}\left(x, z, \alpha_{0}+\beta\right)=\Psi_{L}\left(x, z, \alpha_{0}-\beta\right)$ for $(x, z) \in \mathbf{R}^{n+1}$ and $\beta \in \bigwedge_{n}\left(\mathbf{R}^{n+1}\right)$ with $\beta \cdot \alpha_{0}=0$, where $\alpha_{0}=e_{1} \wedge \cdots \wedge e_{n}$.

(b) The restriction of $\Psi_{L}$ to $\mathbf{R}^{n+1} \times \wedge_{n}\left(\mathbf{R}^{n+1}\right) \cap\left\{\alpha: \alpha \cdot \alpha_{0} \neq 0\right\}$ is of class 2 .

Furthermore, $\Psi_{L}$ is an integrand of class 1 if and only if equality holds in 2.3(F) for every $(x, z, \xi) \in \mathbf{R}^{n+1} \times\left(\mathbf{R}^{n}\right)^{*}$ with $\xi \neq 0$.

Conversely, if $\Psi$ is a convex positive even parametric integrand of degree $n$ on $\mathbf{R}^{n+1}$ which satisfies (a) and (b) then there exists a convex positive nomparametric integrand $L$ of class 2 which satisfies 2.3(A)-(F) and is such that $\Psi=\Psi_{L}$. Further, ellipticity of $\Psi$ [F1, 5.1.2] implies that $L$ is strictly convex.

Proof. That $\Psi_{L}$ is even and satisfies (a) and (b) follows from its definition and 2.3(A). Since

$$
|\alpha|^{2}=|p(\alpha)|^{2}+\mid p\left(\left.\alpha\llcorner d z)\right|^{2} \quad \text { for } \alpha \in \bigwedge_{n}\left(\mathbf{R}^{n+1}\right),\right.
$$

it follows from 2.3 (B) that $\Psi_{L}$ is positive. Further, since for each $(x, z) \in \mathbf{R}^{n+1}$, $\Psi_{L}(x, z, \cdot)$ is the composition of $\Lambda(x, z, \cdot, \cdot)$ with a linear function, convexity of $\Psi_{L}(x, z, \cdot)$ is implied by $4.2(\mathrm{i})$. Finally, if equality holds in $2.3(\mathrm{~F})$ for every $\xi \neq 0$ we infer from 4.2 that $\Lambda$ is continuously differentiable on $\mathbf{R}^{n+1} \times\left[\left(\mathbf{R}^{n}\right)^{*} \times \mathbf{R} \sim\right.$ $\{(0,0)\}]$ hence conclude that $\Psi_{L}$ is an integrand of class 1 .

Let $\Psi$ be a parametric integrand of degree $n$ on $\mathbf{R}^{n+1}$. With $\Psi$ one associates a nonparametric integrand $L_{\Psi}$ on $\mathbf{R}^{n}$ defined as follows: To each $\xi \in\left(\mathbf{R}^{n}\right)^{*}$ correspond

$$
\alpha(\xi)=\alpha_{0}+(-1)^{n+1} \mathbf{D}^{1} \xi \wedge e_{n+1} \in \bigwedge_{n}\left(\mathbf{R}^{n+1}\right)
$$

and define

$$
L_{\Psi}(x, z, \xi)=\Psi(x, z, \alpha(\xi)) \quad \text { for }(x, z) \in \mathbf{R}^{n+1} .
$$

$L_{\Psi}$ is clearly of class 2 if $\Psi$ satisfies (b), and $L_{\Psi}$ satisfies $2.3(\mathrm{~A})$ if $\Psi$ satisfies (a).

If $\Psi$ is positive then homogeneity of $\Psi(x, z, \cdot)$ implies the existence for each compact $K \subset \mathbf{R}^{n+1}$ of $c_{K}>0$ such that

$$
\Psi(x, z, \alpha) \geqslant c_{K}|\alpha| \quad \text { for }(x, z) \in K ;
$$

since $|\boldsymbol{\alpha}(\xi)|=A(\xi)$ this implies (B). Additionally, if $\Psi$ satisfies (b) then homogeneity implies that for $(x, z) \in \mathbf{R}^{n+1}$ and $\alpha \in \bigwedge_{n}\left(\mathbf{R}^{n+1}\right)$ with $\alpha \cdot \alpha_{0} \neq 0$,

$$
D_{\alpha}^{2} \Psi(x, z, \alpha)(\alpha, \beta)=0 \quad \text { whenever } \beta \in \bigwedge_{n}\left(\mathbf{R}^{n+1}\right) \text {. }
$$

This implies the existence of $C_{K}$ such that for $(x, z) \in K$ and $\beta, \gamma \in \wedge_{n}\left(\mathbf{R}^{n+1}\right)$

$$
\left|D_{\alpha}^{2} \Psi(x, z, \alpha)(\beta, \gamma)\right| \leqslant \frac{C_{K}}{|\alpha|}\left[|\beta|^{2}-\frac{(\alpha \cdot \beta)^{2}}{|\alpha|^{2}}\right]^{1 / 2}\left[|\gamma|^{2}-\frac{(\alpha \cdot \gamma)^{2}}{|\alpha|^{2}}\right]^{1 / 2} \text {. }
$$

Inasmuch as

$$
D_{\xi}^{2} L_{\Psi}(x, z, \xi)(\eta, \zeta)=D_{\alpha}^{2} \Psi(x, z, \boldsymbol{\alpha}(\xi))(D \boldsymbol{\alpha}(\eta), D \boldsymbol{\alpha}(\zeta)) \quad \text { for } \xi, \eta, \zeta \in\left(\mathbf{R}^{n}\right)^{*},
$$

we conclude that $L_{\Psi}$ satisfies 2.3(C), (D), (E). Finally, the reverse of the above inequality also holds (with $C_{K}$ replaced by a suitable constant $C_{K}^{\prime} \geqslant 0$ ). Thus 
convexity of $\Psi$ implies convexity of $L_{\Psi}$; moreover, ellipticity of $\Psi$ implies that $C_{K}^{\prime}>0$ for each $K$ (see [F1, 5.1.2 and 5.1.3]) hence $L_{\Psi}$ is strictly convex.

Next we suppose $\Psi$ to be even and verify that $\Psi_{L_{\Psi}}=\Psi$; in view of their continuity and homogeneity it will suffice to show that both functions take the same value on $\alpha \in \bigwedge_{n}\left(\mathbf{R}^{n+1}\right)$ with $\alpha \cdot \alpha_{0}=\mathbf{D}_{n} p(\alpha)=1$. However, this follows from the definition of $\Psi_{L_{\psi}}(\alpha)$ and the observation that

$$
\begin{aligned}
\alpha \circ \xi(\alpha) & =\alpha\left[\mathbf{D}_{n-1} p(\alpha\llcorner d z)]\right. \\
& =\alpha_{0}+(-1)^{n+1}\left(\alpha\llcorner d z) \wedge e_{n+1}=\alpha\right.
\end{aligned}
$$

because $\mathbf{D}^{1} \circ \mathbf{D}_{n-1}$ is the identity map of $\bigwedge_{n-1}\left(\mathbf{R}^{n}\right)$.

Suppose $\Psi$ is convex, positive and even, and satisfies (a) and (b). Then $L=L_{\Psi}$ is convex, positive, of class 2 and satisfies $2.3(\mathrm{~A})-(\mathrm{E})$. In order to verify (F) observe that since $\Psi=\Psi_{L}$,

$$
\Psi\left(x, z, \varepsilon \alpha_{0}+\beta\right)=\Lambda\left(x, z, D_{n-1} p(\beta L d z), \varepsilon\right)
$$

for $\varepsilon>0$ and $\beta \cdot \alpha_{0}=0$; fixing $0 \neq \xi \in\left(\mathbf{R}^{n}\right)^{*}$ and setting $\beta=\alpha(\xi)-\alpha_{0}$ we have

$$
\mathbf{D}_{n-1} p\left(\beta\llcorner d z)=(-1)^{n+1} \mathbf{D}_{n-1} p\left(\mathbf{D}^{1} \xi \wedge e_{n+1}\llcorner d z)=\xi\right.\right.
$$

and so

$$
D_{\varepsilon} \Lambda(x, z, \xi, \varepsilon)=\frac{\partial}{\partial \varepsilon} \Psi\left(x, z, \varepsilon \alpha_{0}+\beta\right) .
$$

Now (a) and the assumption that $\Psi$ is even imply that

$$
\frac{\partial}{\partial \varepsilon} \Psi\left(x, z,-\varepsilon \alpha_{0}+\beta\right)=-\frac{\partial}{\partial \varepsilon} \Psi\left(x, z, \varepsilon \alpha_{0}+\beta\right),
$$

hence convexity of $\Psi$ implies that

$$
\begin{aligned}
0 & \leqslant \lim _{\varepsilon \rightarrow 0^{+}} \frac{\partial}{\partial \varepsilon} \Psi\left(x, z, \varepsilon \alpha_{0}+\beta\right)-\lim _{\varepsilon \rightarrow 0^{-}} \frac{\partial}{\partial \varepsilon} \Psi\left(x, z, \varepsilon \alpha_{0}+\beta\right) \\
& =2 \lim _{\varepsilon \rightarrow 0^{+}} \frac{\partial}{\partial \varepsilon} \Psi\left(x, z, \varepsilon \alpha_{0}+\beta\right) .
\end{aligned}
$$

Referring to equation (4) in the proof of 4.2 we conclude that $L$ satisfies (F). In addition we see that if $\Psi \mid \mathbf{R}^{n+1} \times\left(\bigwedge_{n}\left(\mathbf{R}^{n+1}\right) \sim\{0\}\right)$ is of class 1 then

$$
\lim _{\varepsilon \rightarrow 0^{+}} D_{\varepsilon} \Lambda(x, z, \xi, \varepsilon)=0 ;
$$

that is, equality holds in $(F)$ for $\xi \neq 0$.

\section{Minimizing currents.}

5.1. LEMMA. Let $O$ be an open subset of $\mathbf{R}^{n+1}$ and $C$ be a closed subset of the closure of $O$ such that for each $i=1,2, \ldots$ there exist open sets $O_{i}, V_{i}$ with

$$
\begin{aligned}
& \text { Closure } O_{i} \subset O \subset \text { Closure } O \subset V_{i}, \\
& O_{i} \subset O_{i+1}, \quad O \cap C=\bigcup_{j=1}^{\infty} O_{i} \cap C,
\end{aligned}
$$

and an isotopic deformation of class 1

$$
\eta_{i}: I_{i} \times V_{i} \rightarrow V_{i}
$$


with the following properties: $I_{i}$ is an open interval, $0 \in I_{i}, \eta_{i, 0}$ is the identity, and for each $0<t \in I_{i}, \eta_{i, t}$ is a diffeomorphism of class 1 of $V_{i}$ for which

$$
\eta_{i, t} \mid O_{i}=1_{O} \text { and } \eta_{i, t}(C) \subset O \text {. }
$$

Suppose $T \in \mathscr{R}_{k}\left(\mathbf{R}^{n+1}\right)$ with $\|T\|\left(\mathbf{R}^{n+1} \sim O\right)=0$ and assume $T \mid \mathscr{D}^{k}(O) \in$ $\mathcal{R}_{k}^{\text {loc }}(O)$ to be absolutely (respectively homologically) $\Psi$ minimizing with respect to $O$ where $\Psi$ is a convex nonnegative parametric integrand of degree $k$ on $\mathbf{R}^{n+1}$. If spt $T \subset C$ then $T$ is absolutely (homologically) $\Psi$ minimizing with respect to $C$.

Proof. Assuming $T \mid \mathscr{Q}^{k}(O)$ to be absolutely minimizing we first consider the case where spt $T \subset O$. Denote

$$
\mu=\inf \left\{\int_{T+X} \Psi: X \in \mathscr{Z}_{k}(C) \cap \Re_{k}\left(\mathbf{R}^{n+1}\right)\right\},
$$

and assume $\mu<\int_{T} \Psi$. Choose $X$ and $i$ such that

$$
(1+1 / i) \int_{T+X} \Psi<\int_{T} \Psi, \quad \text { spt } T \subset O_{i} .
$$

Referring to [F1, 5.1.1 and 5.1.7] we see that

$$
\int_{\eta_{i, t \#}(T+X)} \Psi=\int_{T+X} \eta_{i, t}^{\#} \Psi
$$

is a continuous function of $t \in I_{i}$; choose $t>0$ so that

$$
\int_{\eta_{i, t+}(T+X)} \Psi \leqslant(1+1 / i) \int_{T+X} \Psi .
$$

But $\eta_{i, t \#}(T+X)=T+\eta_{i, t \#} X$ hence

$$
\int_{T} \Psi \leqslant \int_{\eta_{i, t *}(T+X)} \Psi<\int_{T} \Psi
$$

Turning to the general case we note that $T_{i}=T\left\llcorner O_{i}\right.$ is $\Psi$ minimizing with respect to $C$ for each $i=1,2, \ldots$, hence if $X \in \mathscr{Z}_{k}(C) \cap \Re_{k}\left(\mathbf{R}^{n+1}\right)$ then

$$
\int_{T_{i}} \Psi \leqslant \int_{T_{i}+X} \Psi<\int_{T+X} \Psi+\int_{T-T_{i}} \Psi
$$

by [F1, 5.1.1]. Thus since $\mathbf{M}\left(T-T_{i}\right) \rightarrow 0$ as $i \rightarrow \infty$, the lower semicontinuity of $\int \Psi$ $[\mathbf{F 2}, 2.3]$ implies that $\int_{T} \Psi \leqslant \int_{T+X} \Psi$.

5.2. Lemma. Assume 2.4 to hold; define $\Psi_{L}$ as in 4.3. Let $f_{0}: D \rightarrow \mathbf{R}$ be locally Lipschitzian with $\left|f_{0}(x)-f(x)\right|<\delta_{0}$ for $x \in D$. Denote $T=\Gamma_{f_{0} \#}\left(\mathbf{E}^{n} \mid \mathscr{D}^{n}(D)\right) \in$ $\Re_{n}^{\text {loc }}(\Omega)$.

(i) Whenever $\nu_{L}(x, z)$ exists

$$
\left\langle\alpha, \mathbf{D}_{1} \nu_{L}(x, z)\right\rangle \leqslant \Psi_{L}(x, z, \alpha) \text { for } \alpha \in \bigwedge_{n}\left(\mathbf{R}^{n+1}\right) .
$$

(ii) If $f_{0}=f$ then whenever $d f(x)$ exists

$$
\left\langle\vec{T}, \mathbf{D}_{1} \nu_{L}\right\rangle(x, f(x))=\Psi_{L}(x, f(x), \vec{T}(x, f(x))) .
$$


(iii) Whenever $d f_{0}(x)$ exists

$$
\Psi_{L}\left(x, f_{0}(x), \vec{T}\left(x, f_{0}(x)\right)\right) A_{f_{0}}(x)=L_{f_{0}}(x) .
$$

(iv) $\int_{D} L_{f_{0}} d \mathcal{L}^{n}=\int_{T} \Psi_{L}$.

Proof. Suppose $p(\alpha) \neq 0$ and $\nu_{L}(x, z)$ exists. Denoting $\alpha_{0}=e_{1} \wedge \cdots \wedge e_{n}$ and $\chi=p^{\#}\left(d x^{1} \wedge \cdots \wedge d x^{n}\right)$ we write $\alpha=a \alpha_{0}+\beta \wedge e_{n+1}$ and, referring to [F1, 1.5.2], compute

$$
\begin{aligned}
\left\langle\alpha, \mathbf{D}_{1} \nu_{L}(x, z)\right\rangle & \left.=(-1)^{n}\left\langle\nu_{L}(x, z), \alpha\right\lrcorner(\chi \wedge d z)\right\rangle \\
& =\left\langle\nu_{L}(x, z), a d z-\left(\left(\beta \wedge e_{n+1}\right)\lfloor d z)\right\lrcorner x\right\rangle \\
& =\left\langle\nu_{L}(x, z), d z-\xi(\alpha)\right\rangle \mathbf{D}_{n} p(\alpha) .
\end{aligned}
$$

Thus by 4.1(ii)

$$
\left|\left\langle\alpha, \mathbf{D}_{1} \nu_{L}(x, z)\right\rangle\right| \leqslant L(x, z, \xi(\alpha))\left|\mathbf{D}_{n} p(\alpha)\right|=\Psi_{L}(x, z, \alpha) ;
$$

(i) now follows from continuity of $\Psi_{L}$.

Next assume $d f_{0}(x)$ exists. From (5) in the proof of 3.2 it follows that

$$
A_{f_{0}}(x) \vec{T}\left(x, f_{0}(x)\right)=\alpha_{0}+(-1)^{n+1}\left(\alpha_{0} L p^{\#} d f_{0}(x)\right) \wedge e_{n+1}
$$

hence

$$
\begin{aligned}
& \mathbf{D}_{n} p\left(\vec{T}\left(x, f_{0}(x)\right)\right)=A_{f_{0}}(x)^{-1} \text { and } \\
& \xi\left(\vec{T}\left(x, f_{0}(x)\right)\right)=A_{f_{0}}(x) p\left[\left(\vec{T}\left(x, f_{0}(x)\right)\llcorner d z)\right\lrcorner x\right] \\
&=\left(e_{1} \wedge \cdots \wedge e_{n}\left\llcorner d f_{0}(x)\right)\right\lrcorner d x^{1} \wedge \cdots \wedge d x^{n} \\
&=\mathbf{D}_{n-1} \mathbf{D}^{1} d f_{0}(x)=d f_{0}(x),
\end{aligned}
$$

which imply (iii).

To obtain (iv) use the area formula [F2, 3.2.3] to compute

$$
\int_{T} \Psi_{L}=\int_{D} \Psi_{L}\left(x, f_{0}(x), \vec{T}\left(x, f_{0}(x)\right)\right) A_{f_{0}}(x) d \mathscr{L}^{n} x
$$

and apply (iii).

Finally, to verify (ii) set $\alpha=\vec{T}(x)$ in (1) and apply (3), 3.1(i), (2) and (iii).

5.3. THEOREM. Assume 2.4 and 2.5 to hold; define $\Psi_{L}$ as in 4.3.

(i) There exists a unique $T_{f} \in \Re_{n}\left(\mathbf{R}^{n+1}\right)$ such that

$$
\begin{gathered}
T_{f} \mid \mathscr{Q}^{n}(D \times \mathbf{R})=\Gamma_{f \#}\left(\mathbf{E}^{n} \mid \mathscr{Q}^{n}(D)\right) \text { and } \\
\left\|T_{f}\right\|\left(\mathbf{R}^{n+1} \sim D \times \mathbf{R}\right)=0 .
\end{gathered}
$$

(ii) Fix $\rho_{1} \in\left(0, \rho_{0}\right)$ and denote

$$
C_{1}=\left\{(x, F(x, \rho)):(x, \rho) \in D \cup B \times\left[-\rho_{1}, \rho_{1}\right]\right\} .
$$

If $C$ is a closed subset of $C_{1}$ with spt $T_{f} \subset C$ then $T_{f}$ is absolutely $\Psi_{L}$ minimizing with respect to $C$.

Proof. Denote $T=\Gamma_{f \#}\left(E^{n} \mid \mathscr{O}^{n}(D)\right)$. Note that by $4.4 \Psi_{L}$ is a convex positive even integrand. With the aid of 5.2 and 3.2 we infer that $\int_{T} \Psi_{L}<\infty$ hence 
$\mathbf{M}(T)<\infty$. Using this one employs standard arguments to complete the proof of (i).

In order to verify (ii) we first adapt the method used in the proof of [F1, 5.4.18] to show that $T$ is absolutely $\Psi_{L}$ minimizing with respect to $\Omega$. Consider $X \in \Re_{n}(\Omega)$ such that $\partial X=0$. Then there exists $S \in \Re_{n+1}(\Omega)$ such that $\partial S=X$. Indeed, $X=\partial H_{\#}([0,1] \times X)$ where $H$ is the homotopy defined by the formula

$$
H(t, x, z)=(x, t z+(1-t) f(x)) \text { for }(t, x, z) \in \mathbf{R} \times \Omega
$$

because $H_{0 \#} X$ is an $n$-dimensional cycle with compact support in $\Gamma_{f}(D)$, which is homeomorphic to $D$.

Choose smoothing functions $\phi^{e}, \varepsilon>0$, for $\mathbf{R}^{n+1}$ (see, for example, [F1, 4.1.2]). Represent $S=\mathbf{E}^{n+1}\left\llcorner\eta\right.$ where $\eta: \mathbf{R}^{n+1} \rightarrow \mathbf{R}$ is $\mathcal{L}^{n+1}$ integrable, and denote $\eta^{e}=$ $\eta * \phi^{e}$. Choose $\Delta_{i}$ and $Q_{i}, i=1,2, \ldots$, as in the proof of 3.2, fix $i$, choose the numbers $z_{1}, z_{2}, \ldots$ as in the proof of 3.2 with $f=f_{1}$, denote $\nu_{L}^{e}=\nu_{L} * \phi^{e}$, and apply equation (6) in the proof of 3.2 with $f_{0}$ replaced by $f+z_{j}$ to obtain

$$
\Gamma_{f+z_{j} \#} Q_{i}\left(\mathbf{D}_{1} \nu_{L}\right)=\int_{\Delta_{i}}\left\langle\nu_{L}^{e}\left(x, f(x)+z_{j}\right),(-d f(x), 1)\right\rangle d L^{n} x .
$$

Applying Lebesgue's bounded convergence theorem, 3.1(i) and 5.2(iv) we conclude that

$$
\lim _{i \rightarrow \infty} \lim _{j \rightarrow \infty} \lim _{\varepsilon \rightarrow 0^{+}} \Gamma_{f+z_{j} \#} Q_{i}\left(\mathbf{D}_{1} \nu_{L}^{e}\right)=\int_{T} \Psi_{L}
$$

On the other hand, referring to (3) in the proof of 3.2 with $S$ replacing $h_{\#}\left([0,1] \times Q_{i}\right)$ we see that

$$
X\left(\mathrm{D}_{1} \nu_{L}^{\varepsilon}\right)=0 \text { for } \varepsilon>0
$$

Fixing $i, j, \varepsilon$ we denote

$$
\begin{aligned}
Y & =\Gamma_{f \#} Q_{i}+X, \\
Y_{j} & =\tau_{\left(0, z_{j}\right) \#} Y=\Gamma_{f+z_{j} \#} Q_{i}+\tau_{\left(0, z_{j}\right) \#} X, \\
Y_{j}^{e} & =Y_{j} * \phi^{e},
\end{aligned}
$$

and infer using 5.2(i) and [F2, 2.5] that

$$
Y_{j}\left(\mathrm{D}_{1} \nu_{L}^{e}\right)=Y_{j}^{e}\left(\mathrm{D}_{1} \nu_{L}\right)<\int_{Y_{j}^{e}} \Psi_{L}
$$

hence

$$
\underset{\varepsilon \rightarrow 0^{+}}{\lim \sup _{j}} Y_{j}\left(\mathrm{D}_{1} \nu_{L}^{\varepsilon}\right)<\int_{Y_{j}} \Psi_{L}
$$

Further, the first proposition of $[$ F1, 5.1.1] implies that

$$
\int_{Y_{j}} \Psi_{L}=\int_{Y} \tau_{\left(0, z_{j}\right)}^{\#} \Psi_{L}=\int \Psi_{L}\left(x, z+z_{j}, \vec{Y}(x, z)\right) d\|Y\|(x, z)
$$

whence we conclude that

$$
\underset{j \rightarrow \infty}{\limsup } \limsup _{\varepsilon \rightarrow 0^{+}} Y_{j}\left(\mathbf{D}_{1} \nu_{L}^{\varepsilon}\right)<\int_{Y} \Psi_{L}
$$


Finally we observe that since $\mathbf{M}\left(T-\Gamma_{f \#} Q_{i}\right) \rightarrow 0$ as $i \rightarrow \infty$,

$$
\lim _{i \rightarrow \infty} \int_{\Gamma_{f *} Q_{i}+X} \Psi_{L}=\int_{T+X} \Psi_{L}
$$

referring to (1), (2), (3) we conclude that $\int_{T} \Psi_{L} \leqslant \int_{T+X} \Psi_{L}$. Consequently, $T$ is absolutely $\Psi_{L}$ minimizing with respect to $\Omega$.

To complete the proof we apply 5.1 to $T_{f}$ with $0=\Omega, V_{i}=U_{i} \times \mathbf{R}$,

$$
\begin{gathered}
O_{i}=D_{i} \times \mathbf{R} \cap\left\{(x, z): F_{-r_{i}}(x)<z<F_{r_{i}}(x)\right\}, \quad r_{i}=\rho_{0} i /(i+1), \\
\eta_{i}(t, x, z)=\left(h_{i}(t, x), z\right)
\end{gathered}
$$

(see 2.5). In order to verify that $\eta_{i, t}(C) \subset \Omega$ it will suffice to show that for $x \in D \cup B$

$$
\left(x_{t}, F_{\rho_{1}}(x)\right) \in \Omega \quad \text { and } \quad\left(x_{t}, F_{-\rho_{1}}(x)\right) \in \Omega, \quad x_{t}=h_{i, t}(x) .
$$

For this purpose we fix $\rho_{2} \in\left(\rho_{1}, \rho_{0}\right)$ and denote by $\delta$ the smaller of the positive numbers $\inf _{D \cup B}\left(F_{\rho_{2}}-F_{\rho_{1}}\right)$, inf ${ }_{D \cup B}\left(F_{-\rho_{1}}-F_{-\rho_{2}}\right)$. Since $F_{\rho_{1}}$ and $F_{-\rho_{1}}$ are uniformly continuous, for each $i$ there exists $t_{i}>0$ such that

$$
\left|F_{\rho_{1}}(x)-F_{\rho_{1}}\left(x_{t}\right)\right|<\delta, \quad\left|F_{-\rho_{1}}(x)-F_{-\rho_{1}}\left(x_{t}\right)\right|<\delta
$$

whenever $x \in D \cup B$ and $|t|<t_{i}$; for such $t$ there clearly exist $\rho^{+}, \rho^{-} \in\left[-\rho_{2}, \rho_{2}\right]$ for which

$$
F_{\rho_{1}}(x)=F_{\rho^{+}}\left(x_{t}\right), \quad F_{-\rho_{1}}(x)=F_{\rho^{-}}\left(x_{t}\right) .
$$

\section{Invariance of solutions.}

6.1. Let $G$ and $U$ be as in $\S 1$, and assume $L$ to be $G$-invariant.

For each $x \in U$ denote

$$
\mathcal{V}_{x}^{*}=\left(\mathbf{R}^{n}\right)^{*} \cap\left\{\xi: \mathbf{T}_{x}(G(x)) \subset \operatorname{ker} \xi\right\}
$$

the annihilator of $T_{x}(G(x))$. Note that

$$
\mathfrak{V}_{g(x)}^{*}=D g(x)^{-1 * \mathscr{V}_{x}^{*}} \quad \text { for } g \in G .
$$

We assume that whenever $x$ lies on a principal orbit $[\mathbf{B}, 2.2]$ and $z \in \mathbf{R}$ there exists a linear map $\Pi_{(x, z)}^{*}:\left(\mathbf{R}^{n}\right)^{*} \rightarrow \mathcal{V}_{x}^{*}$ such that the restriction of $\Pi_{(x, z)}^{*}$ to $\mathcal{V}_{x}^{*}$ is the identity and

$$
L\left(x, z, \Pi_{(x, z)}^{*}(\xi)\right) \leqslant L(x, z, \xi) \quad \text { for } \xi \in\left(\mathbf{R}^{n}\right)^{*},
$$

with strict inequality for $\xi \notin \mathfrak{V}_{x}^{*}$. We also assume that $\Pi_{(x, z)}^{*}(\xi)$ is continuous as a function of $(x, z, \xi)$ and is $G$-invariant in the sense that

$$
D g(x)^{*} \circ \Pi_{(g(x), z)}^{*}=\Pi_{(x, z)}^{*} \circ D g(x)^{*} \quad \text { for } g \in G .
$$

A large class of $G$-invariant integrands satisfying the above conditions can be obtained in the following way: Let $\mu$ be a $G$-invariant Riemannian metric on $U$. (Such a metric can be obtained by averaging the standard inner product of $\mathbf{R}^{n}$ with respect to the action of $G$.) Extending $\mu$ to covectors in the usual way so that the dual of an orthonormal basis is orthonormal we denote the corresponding norms on $\left(\mathbf{R}^{n}\right)^{*}$ by $\|\xi\|_{\mu(x)}$. Suppose $L$ can be expressed in the form $L(x, z, \xi)=$ $\mathcal{L}\left(x, z,\|\xi\|_{\mu(x)}\right)$, where $\mathcal{L}: \mathbf{R}^{n} \times \mathbf{R} \times \mathbf{R} \rightarrow \mathbf{R}$ is of class 1 and $\mathcal{L}(x, z, \cdot)$ is strictly 
increasing for each $(x, z) \in U \times \mathbf{R}$. Then the map $\Pi_{(x, z)}^{*}$ can be taken to be orthogonal projection onto $\mathcal{V}_{x}^{*}$; the invariance property is implied by those of $\mu(x)$ and $\mathfrak{V}_{x}^{*}$. Finally, we note that for such $L, G$-invariance reduces to

$$
L(g(x), z, \xi) J g(x)=L(x, z, \xi) \quad \text { for }(x, z, \xi) \in U \times \mathbf{R} \times\left(\mathbf{R}^{n}\right)^{*} .
$$

The action of $G$ on $U$ induces an action of $G$ on $U \times \mathbf{R}$ in the obvious way:

$$
g(x, z)=(g(x), z) \text { for } g \in G,(x, z) \in U \times \mathbf{R} .
$$

The orbit $G(x, z)=G(x) \times\{z\}$, and principal orbits in $U \times \mathbf{R}$ correspond to those in $U$. Each two principal orbits are diffeomorphic; denote their dimension by $m$. Whenever $x$ lies on a principal orbit we choose $0 \neq \alpha \in \bigwedge_{m} T_{x}(G(x))$ and, regarding $\alpha \in \bigwedge_{m}\left(\mathbf{R}^{n+1}\right)$, denote

$$
\mathscr{V}_{x}=\left\{\alpha \wedge \beta: \beta \in \wedge_{n-m}\left(\mathbf{R}^{n+1}\right)\right\}
$$

$\mathcal{V}_{x}$ is clearly independent of the choice of $\alpha$ and is a linear subspace of $\wedge_{n}\left(\mathbf{R}^{n+1}\right)$ of dimension $n+1-m$.

6.2. Lemma. Assume 2.4 to hold; define $\Psi_{L}$ as in 4.3 .

(i) $\Psi_{L}$ is invariant under the action of $G$.

(ii) Let $(x, z) \in U \times \mathbf{R}$ lie on a principal orbit. Then there exists a linear map $\Pi_{(x, z)}: \bigwedge_{n}\left(\mathbf{R}^{n+1}\right) \rightarrow \mathfrak{V}_{x}$ such that the restriction of $\Pi_{(x, z)}$ to $\mathfrak{V}_{x}$ is the identity and

$$
\Psi_{L}\left(x, z, \Pi_{(x, z)}(\alpha)\right) \leqslant \Psi_{L}(x, z, \alpha) \text { for } \alpha \in \bigwedge_{n}\left(\mathbf{R}^{n+1}\right)
$$

with strict inequality for $\alpha \notin \mathfrak{V}_{x}$ with $p(\alpha) \neq 0$. Further, $\Pi_{(x, z)}(\alpha)$ is continuous as $a$ function of $(x, z, \alpha)$ and is invariant in the sense that

$$
\Pi_{g(x, z)} \circ \bigwedge_{n} D g(x, z)=\bigwedge_{n} D g(x, z) \circ \Pi_{(x, z)} \text { for } g \in G .
$$

Proof. Consider $g \in G$ and $\alpha \in \bigwedge_{n}\left(\mathbf{R}^{n+1}\right)$ with $p(\alpha) \neq 0$, and fix $(x, z) \in U$. Inasmuch as

$$
(\operatorname{det} \gamma) \gamma^{-1 *}\left(\mathbf{D}_{n-1} \beta\right)=\mathbf{D}_{n-1} \bigwedge_{n-1} \gamma(\beta) \text { for } \beta \in \wedge_{n-1}\left(\mathbf{R}^{n}\right)
$$

and $\wedge_{n} \gamma(p(\alpha))=(\operatorname{det} \gamma) p(\alpha)$ whenever $\gamma$ is an isomorphism of $\mathbf{R}^{n}$, we have

$$
\xi\left(\wedge_{n} D g(x, z)(\alpha)\right)=D g(x)^{-1} * \xi(\alpha) \text {. }
$$

Consequently, the $g$-invariance of $L$ implies that

$$
\Psi_{L}\left(g(x, z), \wedge_{n} D g(x, z)(\alpha)\right)=\Psi_{L}(x, z, \alpha),
$$

and equality for arbitrary $\alpha$ follows from continuity of $\Psi_{L}$. This proves (i).

Next assume $x$ to lie on a principal orbit and denote

$$
\mathfrak{V}_{x}^{\prime}=\bigwedge_{n}\left(\mathbf{R}^{n+1}\right) \cap\left\{\alpha: \mathbf{D}_{n-1} p\left(\alpha\llcorner d z) \in \mathscr{V}_{x}^{*}\right\}\right.
$$

$\mathcal{V}_{x}^{\prime}$ is clearly a linear subspace of $\bigwedge_{n}\left(\mathbf{R}^{n+1}\right)$. Suppose $\xi \in \mathscr{V}_{x}^{*}$. Since

$$
\left\langle\mathbf{D}^{1} \xi, \mathbf{D}_{1} v\right\rangle=(-1)^{n-1}\langle v, \xi\rangle \text { for } v \in \mathbf{R}^{n},
$$

we infer that

$$
\mathbf{R}^{n} \cap\left\{v:\left(\mathbf{D}^{1} \xi\right) \wedge v=0\right\}=\operatorname{ker} \xi,
$$

which implies that

$$
\mathbf{D}^{1}\left(\mathfrak{V}_{x}^{*}\right)=\bigwedge_{n-1}\left(\mathbf{R}^{n}\right) \cap\left\{\alpha_{0} \wedge \beta^{\prime}: \beta^{\prime} \in \bigwedge_{n-1-m}\left(\mathbf{R}^{n}\right)\right\}
$$


where $0 \neq \alpha_{0} \in \wedge_{m} \mathbf{T}_{x}(G(x))$. Consequently, $\mathbf{D}_{n-1} p\left(\alpha\llcorner d z) \in \mathfrak{V}_{x}^{*}\right.$ only if

$$
p\left(\alpha\llcorner d z)=\alpha_{0} \wedge \beta^{\prime} \text { for some } \beta^{\prime} \in \wedge_{n-1-m}\left(\mathbf{R}^{n}\right),\right.
$$

whence we infer that $\alpha=\alpha_{0} \wedge \beta$ where $\beta \in \wedge_{n-m}\left(\mathbf{R}^{n+1}\right)$ and $\alpha_{0}$ is regarded as a member of $\Lambda_{m}\left(\mathbf{R}^{n+1}\right)$. From this we conclude that $\mathfrak{V}_{x}^{\prime} \subset \mathfrak{V}_{x}$; equality follows from the observation that $\operatorname{dim} \mathcal{V}_{x}^{\prime}>n+1-m=\operatorname{dim} \mathcal{V}_{x}$.

We now define the projection $\Pi_{(x, z)}$ by the formula

$\Pi_{(x, z)}(\alpha)=(-1)^{n+1}\left[\mathbf{D}^{1} \Pi_{(x, z)}^{*} \mathbf{D}_{n-1} p(\alpha\llcorner d z)] \wedge e_{n+1}+p(\alpha)\right.$ for $\alpha \in \wedge_{n}\left(\mathbf{R}^{n+1}\right)$.

This function is clearly linear and continuous; the invariance property follows from that of $\Pi_{(x, z)}^{*}$ together with (1) and the dual identity

$$
\bigwedge_{n-1} \gamma\left(\mathbf{D}^{1} \xi\right)=(\operatorname{det} \gamma) \mathbf{D}^{1} \gamma^{-1 *}(\xi) \text { for } \xi \in\left(\mathbf{R}^{n}\right)^{*}
$$

Since

$$
\alpha=(-1)^{n+1} p(\alpha \mathrm{L} d z) \wedge e_{n+1}+p(\alpha),
$$

the definition implies that $\Pi_{(x, z)}(\alpha)=\alpha$ in case $\alpha \in \mathscr{V}_{x}$. To verify that $\Pi_{(x, z)}(\alpha) \in$ $\mathcal{V}_{x}$ for each $\alpha$ observe that

$$
\begin{aligned}
\mathbf{D}_{n-1} p\left(\Pi_{(x, z)}(\alpha)\llcorner d z)\right. & =\mathbf{D}_{n-1} \mathbf{D}^{1} \Pi_{(x, z)}^{*} \mathbf{D}_{n-1} p(\alpha\llcorner d z) \\
& =\Pi_{(x, z)}^{*} \mathbf{D}_{n-1} p\left(\alpha\llcorner d z) \in \mathscr{V}_{x}^{*} ;\right.
\end{aligned}
$$

this also implies that if $p(\alpha) \neq 0$ then

$$
\xi \circ \Pi_{(x, z)}(\alpha)=\Pi_{(x, z)}^{*} \circ \xi(\alpha) .
$$

Finally, suppose $\alpha \in \bigwedge_{n}\left(\mathbf{R}^{n+1}\right) \sim \mathcal{V}_{x}$ with $p(\alpha) \neq 0$. Then $\xi(\alpha) \notin \mathcal{V}_{x}^{*}$, hence

$$
\begin{aligned}
\Psi_{L}\left(x, z, \Pi_{(x, z)} \alpha\right) & =L\left(x, z, \xi \circ \Pi_{(x, z)} \alpha\right)|p(\alpha)| \\
& =L\left(x, z, \Pi_{(x, z)}^{*} \circ \xi(\alpha)\right)|p(\alpha)| \\
& <L(x, z, \xi(\alpha))|p(\alpha)|=\Psi_{L}(x, z, \alpha) .
\end{aligned}
$$

6.3. TheOREM. Assume the hypotheses of 2.4, 2.5 and 6.1 to hold. Further, assume that $D \cup B$ is a Lipschitz neighborhood retract, that $B$ is the union of a countable family of submanifolds of class 1 , and that there exists a continuous function $f_{0}$ : $D \cup B \rightarrow \mathbf{R}$ such that

$$
\begin{array}{cc}
\left|f(x)-f_{0}(x)\right|<\delta_{0} / 5 & \text { for } x \in D \cup B \text { and } \\
f_{0} \circ g=f_{0} & \text { for } g \in G .
\end{array}
$$

If $f \circ g|B=f| B$ whenever $g \in G$ then

$$
f \circ g=f \text { for every } g \in G \text {. }
$$

Proof. Smooth $f_{0}$ to obtain $f_{1} \in \mathcal{E}^{0}(U)$ such that

$$
\left|f_{0}(x)-f_{1}(x)\right|<\delta_{0} / 5 \text { for } x \in D \cup B,
$$

and denote by $f_{1 G}$ the average of $f_{1}$ defined by the formula

$$
f_{1 G}(x)=\int_{G} f_{1} \circ g(x) d \mu g,
$$


where $\mu$ is the Haar measure on $G$ with $\mu(G)=1$. Then $f_{1 G} \in \mathcal{E}^{0}(U)$ and is $G$-invariant, and

$$
\left|f_{1 G}(x)-f(x)\right|<2 \delta_{0} / 5 \text { for } x \in D
$$

Denote

$$
K=\left\{(x, z): x \in D \cup B,\left|z-f_{1 G}(x)\right| \leqslant 2 \delta_{0} / 5\right\} .
$$

It is not difficult to verify that $K$ satisfies the requirements placed on the set $C$ in 5.3, hence we infer from 5.3 that $T_{f}$ is absolutely $\Psi_{L}$ minimizing with respect to $K$. This implies that $T_{f}$ is homologically $\Psi_{L}$ minimizing in $K$ with respect to $U \times \mathbf{R}$ in the sense of [B, 2.4]. $K$ clearly satisfies the requirements of $[\mathbf{B}, 2.1]$; we would like to apply [B, 4.4] (with $M=U \times \mathbf{R}$ and $L=\varnothing$ ) to conclude that $T_{f}$ is $G$-invariant. However, although $\Psi_{L}$ is convex, positive and even by 4.4 we are unable to verify that $\Psi_{L}$ is $G$-invariant in the sense of [B, 2.5]. Indeed, for this to be true one would need strict inequality in 6.2(ii) for every $\alpha \notin \mathcal{V}_{x}$, and there is no reason to believe that this is true. On the other hand, in proving [B, 4.4] we used strict inequality only in the proof of Lemma 3.6, and there only as it applies to $\alpha=\vec{T}_{f}(x, z)$. However, because of 5.3(i) we see that $p\left(\vec{T}_{f}(x, z)\right) \neq 0$ for $\left\|T_{f}\right\|$ almost all $(x, z)$, hence conclude that $[B, 3.6]$ and consequently $[B, 4.4]$ can be applied to $T_{f}$ once hypotheses (ii) and (iii) of [B, 4.3] are verified. (ii) is implied by 5.3(i); we obtain the current $T^{\prime}$ as follows:

Choose a $G$-invariant Riemannian metric $\mu$ on $U$ (see 6.1) and denote by $\delta_{\mu}(x)$ the distance from $x \in U$ to $U \sim D$. Using $\delta_{\mu}$ in place of $\delta$ we proceed as in the proof of 3.2 to obtain an increasing sequence of open sets $\Delta_{1}, \Delta_{2}, \ldots$ whose union is $D$ such that for each $i$, closure $\Delta_{i} \subset D$ and

$$
Q_{i} \in \mathbf{I}_{n}\left(\mathbf{R}^{n}\right) \text { where } Q_{i}=\mathbf{E}^{n} \mathrm{~L} \Delta_{i} .
$$

Each $Q_{i}$ is $G$-invariant because $\delta_{\mu} \circ g=\delta_{\mu}$ for $g \in G$. We also define the homotopy $h$ as in the proof of 3.2 with $f_{2}=f$ and $f_{1}$ equal to the average $f_{G}$ of $f$.

Denoting $S_{i}=h_{\#}\left([0,1] \times Q_{i}\right)$ and using $[F 1,4.1 .30]$ and Fubini's theorem we obtain

$$
\mathbf{M}\left(S_{j}-S_{i}\right) \leqslant \int_{0}^{1} \int_{\Delta_{j} \sim \Delta_{i}} J h(t, x) d \mathcal{L}^{n} x d \mathcal{L}^{1} t \text { for } j>i
$$

however, $\operatorname{Jh}(t, x)=\left|f(x)-f_{G}(x)\right|$ hence

$$
\mathbf{M}\left(S_{j}-S_{i}\right) \leqslant \sup _{D}\left|f-f_{G}\right|^{\mathscr{L}^{n}}\left(\Delta_{j} \sim \Delta_{i}\right) .
$$

Therefore there exists $S \in \mathcal{R}_{n+1}(U \times \mathbf{R})$ such that $\mathbf{M}\left(S-S_{i}\right) \rightarrow 0$ as $i \rightarrow \infty$. Inasmuch as $f_{G}|B=f| B, 5.3(\mathrm{i})$ and equations (1) and (2) in the proof of $3.2 \mathrm{imply}$ that

$$
\Gamma_{f_{G} \#} Q_{i} \rightarrow T_{f_{G}}=T_{f}-\partial S \in \mathbf{F}_{n}(U \times \mathbf{R}) \text { as } i \rightarrow \infty .
$$

$T_{f_{G}}$ is invariant because each $\Gamma_{f_{G} \#} Q_{i}$ is invariant, and with the exception of the requirement that $\mathbf{M}\left(T_{f_{G}}\right)<\infty$ the remaining conditions of [B, 4.3(iii)] follow for $T_{f_{G}}$ in a similar way. In case $L(x, z, \xi)$ does not depend on $z$ we can use 5.2, Jensen's inequality [F1, 2.4.19] and 3.2 to verify that 


$$
\int_{T_{f_{G}}} \Psi_{L}=\int_{D} L_{f_{G}} d \stackrel{L}{ }^{n} \leqslant \int_{D} L_{f} d L^{n}<\infty,
$$

which implies that $\mathbf{M}\left(T_{f_{G}}\right)<\infty$; however, in general we are unable to verify this and must proceed as follows:

Denote by $h^{\prime}$ the homotopy defined as in the proof of 3.2 with $f_{1}=f_{G}$ and $f_{2}=f_{1 G}$. Clearly $T_{f_{1 G}}=\Gamma_{f_{1 G} \#}\left(\mathbf{E}^{n}\llcorner D) \in \Re_{n}(U \times \mathbf{R})\right.$; as before there exists $S^{\prime} \in$ $\Re_{n+1}(U \times \mathbf{R})$ such that

$$
\mathbf{M}\left(S^{\prime}-h_{\#}^{\prime}\left([0,1] \times Q_{i}\right)\right) \rightarrow 0 \text { as } i \rightarrow \infty .
$$

We infer with aid of (1) and (2) in the proof of 3.2 that

$$
h_{\#}^{\prime}\left([0,1] \times \partial Q_{i}\right) \rightarrow T_{f_{1 G}}-T_{f_{G}}-\partial S^{\prime} \in \mathbf{F}_{n}(U \times \mathbf{R}) \quad \text { as } i \rightarrow \infty
$$

hence $\mathbf{M}\left(T_{f_{1 G}}-T_{f_{G}}-\partial S^{\prime}\right)<\infty$; therefore,

$$
T^{\prime}=\partial S^{\prime}+T_{f_{G}} \in \mathbf{F}_{n}(U \times \mathbf{R}) \text { and } \mathbf{M}\left(T^{\prime}\right)<\infty .
$$

( $T^{\prime}$ is in fact rectifiable by $[\mathbf{F 1}, 4.2 .16]$.) Each $h_{\#}^{\prime}\left([0,1] \times Q_{i}\right)$ is clearly invariant hence so are $S^{\prime}$ and $T^{\prime}$. In case $\xi \in \mathfrak{X}_{G}(U \times \mathbf{R})$ with spt $\xi \subset(U \times \mathbf{R})_{0}($ see $[\mathbf{B}, 2.1$ and 2.2]) one verifies directly that $T_{f_{G}} \wedge \xi=0$ and uses [B, 3.3] to infer that $\partial S^{\prime} \wedge \xi=0$; thus $T^{\prime} \wedge \xi=0$. Finally, $T^{\prime}-T_{f}=\partial\left(S^{\prime}-S\right)$ and we conclude that $T^{\prime}$ satisfies the requirements of $[B, 4.3($ iii) $]$.

\section{REFERENCES}

[B] J. E. Brothers, Invariance of solutions to invariant parametric variational problems, Trans. Amer. Math. Soc. 262 (1980), 159-179.

[C] C. Caratheodory, Calculus of variations and partial differential equations of the first order. Part II, Holden-Day, San Francisco, Calif., 1967.

[F1] H. Federer, Geometric measure theory, Springer-Verlag, New York, 1969.

[F2] __ Real flat chains, cochains and variational problems, Indiana Univ. Math. J. 24 (1974), 351-407.

[GT] D. Gilbarg and N. S. Trudinger, Elliptic partial differential equations of second order, SpringerVerlag, New York, 1977.

[H] R. M. Hardt, Uniqueness of nonparametric area minimizing currents, Indiana Univ. Math. J. 26 (1977), 65-71.

[M] C. B. Morrey, Jr., Multiple integrals in the calculus of variations, Springer-Verlag, New York, 1966.

DEPARTMENT OF MATHEMatics, Indiana University, BloOmington, Indiana 47401 